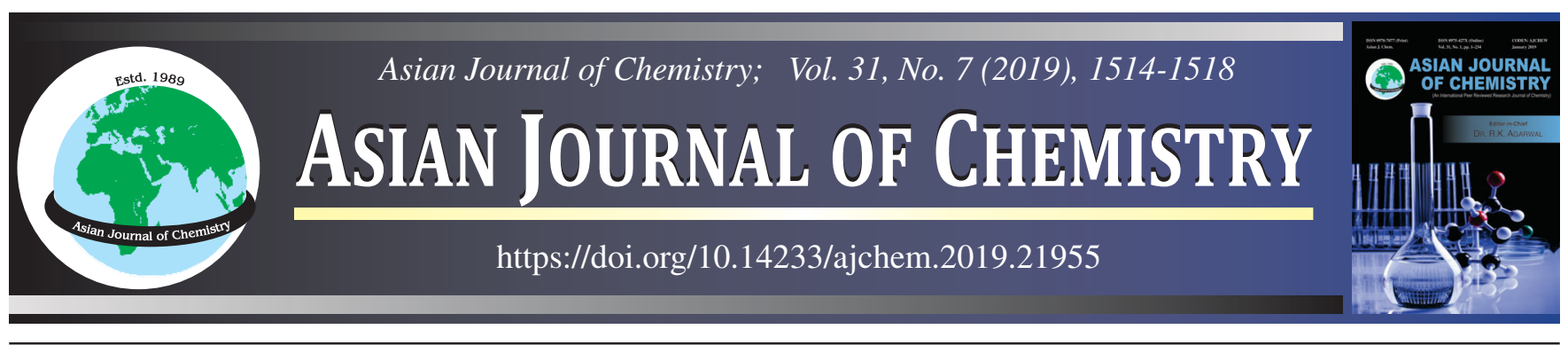

\title{
Isolation and Structural Characterization of Degradation Products of Finasteride by Preparative HPLC, HRMS and 2D NMR
}

\author{
Santhosh Guduru ${ }^{1,2}$, V.V.S.R.N. Anjt Karun Mutha ${ }^{1,2}$, B. Vijayabhaskar ${ }^{1}$, Muralidharan Kaliyaperumal ${ }^{1}$, \\ Prabhakar S. Achanta ${ }^{1}$, Sreenivasa Reddy Anugu ${ }^{1}$, Bharath Komandla ${ }^{1}$, Raghu Babu Korupolu ${ }^{2}$, \\ Kishore Babu Bonige ${ }^{2}$ and Chidananda Swamy Rumalla ${ }^{1, *}$
}

${ }^{1}$ Department of Medicinal Chemistry, G.V.K. Biosciences Pvt. Ltd, IDA Mallapur, Hyderabad-500076, India

${ }^{2}$ Department of Engineering Chemistry, Andhra University, Visakhapatnam-530003, India

*Corresponding author: E-mail: chidanand_swamy@yahoo.co.in

Received: 24 January 2019;

Accepted: 14 March 2019;

Published online: 21 May 2019;

AJC-19406

Finasteride is a 5 - $\alpha$-reductase inhibitor with a steroidal skeleton and an amide group in its structure. It was subjected to forced degradation to observe its stability under stress conditions according to ICH guidelines. It was found to be stable to base and peroxide. However, in acid medium, three degradation products were observed. All of them were isolated from the reaction mixture by preparative HPLC. Their structures were elucidated by extensive analysis of 1D, 2D NMR spectra and HRMS. To best of our knowledge, none of them have been reported elsewhere.

Keywords: Finasteride, LC-MS, NMR, Degradation studies.

ᄂ - - - - - - - - - - - - - - - -

\section{INTRODUCTION}

Finasteride is used to stop the scalp hair loss and enlarged prostrate in men. It is also used to treat excessive hair growth in women and as a part of hormone therapy for transgender women [1,2]. Finasteride has a steroidal skeleton with 4th atom as a nitrogen instead of a carbon making it a 2-quinolone derivative. The structure contains two amides, one as an $\alpha, \beta-$ unsaturated lactam (ring-A) and the other as a tert-butyl amide at $\mathrm{C}-17$.

The stability indicating stress studies are gaining importance given the fact that the drugs are prone to degradation during their shelf life (between the time of manufacturing and consumption by the patient) [3-5]. Therefore, accelerated stress stabilities are recommended by several regulatory authorities including ICH [6-8] for rapid production of degradation products and to test for their toxicity.

The stability of finasteride under stress conditions has not been studied extensively. There are few reports on the HPLC indicated stability studies [9-12] further, there is one report on the UPLC method development of related impurities of finasteride [13].
As far as literature search, this is the first attempt at degradation of finasteride and the isolation of its degradation products. In the present study, the drug was subjected to acid, base and hydrogen peroxide mediated stress conditions as per ICH guidelines. It was found to be stable under both base and peroxide stress conditions. The acid mediated stress resulted in the production of three new degradation products. All of them were isolated and their structures were elucidated by extensive analysis of 1D and 2D NMR and mass spectra. The degradation products were found to be 4-hydroxy-4a,6a-dimethyl2-oxohexadecahydro- $1 H$-indeno[5,4,f]quinoline-7carboxylic acid (DP-1), 4a,6a-dimethyl-2-oxo-2,4a,4b,5,6,6a, 7,8,9,9a,9b, 10,11,11a-tetradecahydro- $1 H$-indeno[5,4-f] quinoline-7carboxamide (DP-2) and 4a,6a-dimethyl-2-oxo-2,4a,4b,5,6,6a, 7,8,9,9a,9b,10,11,11a-tetradecahydro- $1 H$-indeno[5,4-f]quinoline-7-carboxylic acid (DP-3).

\section{EXPERIMENTAL}

Finasteride was obtained as a kind gift sample from an industry (Hyderabad, India). HPLC grade solvents and buffers were used. Water used was Milli-Q grade.

This is an open access journal, and articles are distributed under the terms of the Creative Commons Attribution-NonCommercial-ShareAlike 4.0 (CC BY-NC-SA 4.0) International License which allows readers to freely read, download, copy, distribute, print, search, or link to the full texts of its articles and to use them for any other lawful non-commercial purpose as long as the original source is duly acknowledged. 
The LC-MS data was collected using an Agilent 1290 Infinity LC System (Agilent, Santa Clara, CA, USA) with ACQUITY BEH C18 $(2.1 \times 50 \mathrm{~mm}, 1.7 \mu \mathrm{m}$ particle size $)$ column; column temperature was maintained at $45^{\circ} \mathrm{C}$; Binary pump with mobile phase A: $0.1 \%$ formic acid in water; mobile phase B: $100 \%$ Acetonitrile; T/\% of B: 0.0/2.0, 0.2/2.0, 1/20, 5.0/80, 6.0/98, 8.0/98, 9.0/2, 10/2; Diluent: Mobile phase; flow rate $0.8 \mathrm{~mL} / \mathrm{min}$; Detection: $215 \mathrm{~nm}$.

Preparative HPLC was carried out using Waters quaternary gradient module 2545, Waters photo diode array detector module 2998; Waters Auto sampler 2707; Waters Fraction collector III (Waters Corp., Milford, MA, USA); column: Symmetry C8 $(300 \times 19 \mathrm{~mm}) 7 \mu$, mobile phase A: $0.1 \%$ formic acid (Aq); mobile phase B: Acetonitrile: T \% of B: 0.0/10, $8.0 / 50,11 / 50,11.1 / 98,12 / 98,12.1 / 10,15 / 10$; flow rate: 20 $\mathrm{mL} / \mathrm{min}$.

HRMS data of all the degradation products was acquired with Waters micro mass Q-TOF equipped with an electrospray Ionization (ESI) source. Samples were operated in positive and negative mode to enable detection of degradants. Leucine encephalin $(\mathrm{m} / \mathrm{z}$ : $555.62268 \mathrm{Da})$ was used as reference lock mass calibrant to achieve the typical mass accuracies with Mass Lynx software. The optimal conditions of analysis were as follows: capillary voltage of $3.5 \mathrm{kV}$, sample cone at $25 \mathrm{~V}$ and the scan range from 100 to 2200 Daltons. The source temperature was $120^{\circ} \mathrm{C}$ and the desolvation temperature was set at $350^{\circ} \mathrm{C}$. Nitrogen was used as the nebulization gas at flow rate of $750 \mathrm{~L} / \mathrm{h}$.

1D and 2D NMR spectra of finasteride and its degradation products were recorded in DMSO- $d_{6}$ on $400 \mathrm{MHz}$ Bruker NMR spectrometer. Chemical shift values of ${ }^{1} \mathrm{H}$ and ${ }^{13} \mathrm{C}$ are measured on $\delta$ scale in ppm comparative to tetra methyl silane (TMS) as internal standard. The spectra were referenced to $\delta 0.00 \mathrm{ppm}$ in ${ }^{1} \mathrm{H}$ NMR (TMS) and $\delta 39.50 \mathrm{ppm}$ in ${ }^{13} \mathrm{C}$ NMR $\left(\mathrm{DMSO}-d_{6}\right)$.

Stress methods: Compound finasteride was subjected to stress conditions as per ICH guideline Q1A (R2)5 to identify the potential degradants that could be formed during the longterm storage. The details of the stress methods are shown in Table-1.

\begin{tabular}{cccc}
\multicolumn{4}{c}{ TABLE-1 } \\
\multicolumn{4}{c}{ STRESS CONDITIONS FOR OPTIMUM DEGRADATION } \\
\hline $\begin{array}{c}\text { Stress } \\
\text { condition }\end{array}$ & $\begin{array}{c}\text { Concentration of } \\
\text { stressor }\end{array}$ & $\begin{array}{c}\text { Exposure } \\
\text { condition }\left({ }^{\circ} \mathrm{C}\right)\end{array}$ & Duration $(\mathrm{h})$ \\
\hline \multirow{2}{*}{ Hydrolysis } & Acid $0.5 \mathrm{~N} \mathrm{HCl}$ & 60 & 8 \\
& Base $0.5 \mathrm{~N} \mathrm{NaOH}$ & 60 & 8 \\
Oxidation & $5 \% \mathrm{H}_{2} \mathrm{O}_{2}$ & 60 & 8 \\
\hline
\end{tabular}

\section{RESULTS AND DISCUSSION}

After $8 \mathrm{~h}$ of heating, degradation products were analyzed. $1 \mathrm{~mL}$ of the resultant acid degradation solution was dissolved in mobile phase and a sample of $10 \mu \mathrm{L}$ was subjected to LCMS analysis. Drug solution treated with acid showed three degradants and no degradation of the drug was observed in peroxide as well as base treated drug solution as shown in Fig. 1. Acid treated solutions were taken up for isolation of all the three detected degradants.
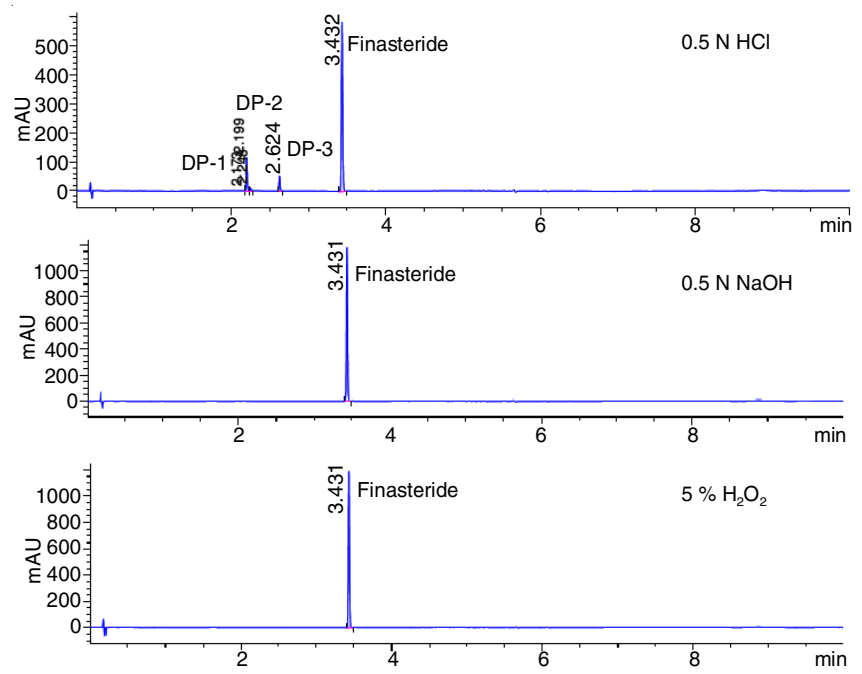

Fig. 1. Acid, base and hydrogen peroxide degradation chromatograms of finasteride drug substance

Isolation of acid degradation products: Preparative HPLC fractions containing degradants under acidic condition were collected and lyophilized. The products were labeled as DP-1, DP-2 and DP-3. The extensive analysis of HRMS and 1D, 2D NMR data confirmed the structures of the degradation products. Literature survey revealed that DP-1, DP-2 and DP3 are novel compounds. Structures of finasteride and its degradation products in various stress conditions are shown in Fig. 2.

Structure elucidation of DP-1: The HRMS showed a protonated molecular ion peak at $m / z 336.2178[\mathrm{M}+\mathrm{H}]^{+}$corresponding to m.f. $\mathrm{C}_{19} \mathrm{H}_{29} \mathrm{NO}_{4}$. The ${ }^{1} \mathrm{H}$ NMR spectrum revealed that DP-1 had one carboxylic acid proton (observed at 11.9 ppm) and one hydroxy proton (observed at $4.92 \mathrm{ppm}$ ). The absence of olefinic and tertiary butyl protons in the NMR of DP-1 suggested hydration of the alkene and hydrolysis of the tertiary butyl amide in the side chain under acidic condition. To ascertain the position of hydroxyl ${ }^{13} \mathrm{C} \mathrm{HMBC}$ was performed and the DP-1 shown in Fig. 3 was found to be the correct structure. In ${ }^{13} \mathrm{C}$ HMBC, H-6 (3.67 ppm) showed correlation with C-2 (169.7 ppm), C-1 (38.2 ppm), C-5 (38.8 ppm), C-4 (52.7 $\mathrm{ppm})$ and $\mathrm{C}-18$ (11.8 ppm) as shown in Fig. 4. H-6 showed correlation with $\mathrm{C}-18$ indicating that hydroxyl group is located on $\mathrm{C}-6$ and not on $\mathrm{C}-1$. The complete assignments of ${ }^{1} \mathrm{H}$ and ${ }^{13} \mathrm{C}$ chemical shift values for DP- 1 are tabulated in Table- 2 . The observed regioselectivity is in line with the established mechanistic prediction of reactivity of $\alpha, \beta$-unsaturated carbonyl compound. The nucleophilic oxygen of water molecule is expected to add to the electrophilic $\beta$ carbon of the lactam.

Structure elucidation of DP-2: The HRMS showed a protonated molecular ion peak at $m / z 317.2232[\mathrm{M}+\mathrm{H}]^{+}$corresponding to m.f. $\mathrm{C}_{19} \mathrm{H}_{28} \mathrm{~N}_{2} \mathrm{O}_{2}$. DP-2 showed all protons that are present in finasteride except tertiary butyl group protons in the side chain. DP-2 indicated primary amide protons (observed at $6.71 \mathrm{ppm}$ and $6.91 \mathrm{ppm}$ ) suggesting the hydrolytic cleavage of the tertiary butyl amide $-\mathrm{CONHC}\left(\mathrm{CH}_{3}\right)_{3}$ to $-\mathrm{CONH}_{2}$. This can be attributed to the ready formation of stable tertiary butyl carbocation under acidic condition. Carbonyl carbon (21-C) of the primary amide was seen at $173.8 \mathrm{ppm}$ in ${ }^{13} \mathrm{C}$ NMR. All proton versus proton correlations in COSY and all proton versus carbon correlations in HSQC and HMBC matched well 


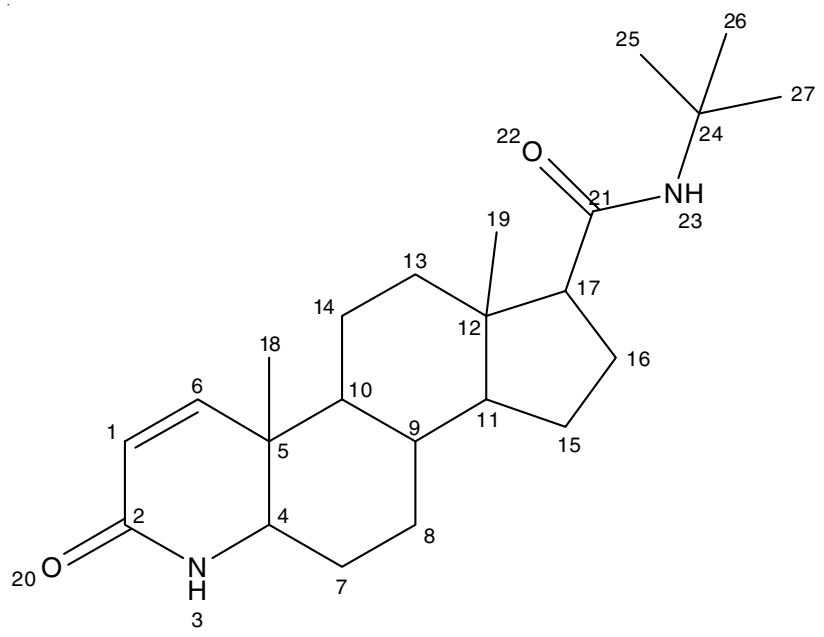

Finasteride

Chemical Formula: $\mathrm{C}_{23} \mathrm{H}_{36} \mathrm{~N}_{2} \mathrm{O}_{2}$

Exact Mass: 372.2777

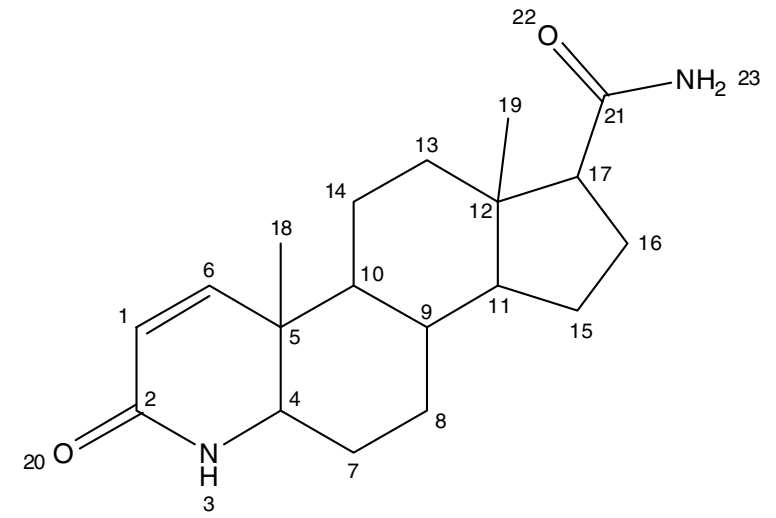

DP-2

Chemical Formula: $\mathrm{C}_{19} \mathrm{H}_{28} \mathrm{~N}_{2} \mathrm{O}_{2}$

Exact Mass: 316.2151<smiles>CC12C(CC[C@@H]1C(=O)O)C1CCC3NC(=O)CC(O)C34C1CCC42C</smiles>

DP-1

Chemical Formula: $\mathrm{C}_{19} \mathrm{H}_{29} \mathrm{NO}_{4}$

Exact Mass: 335.2097

Fig. 2. Structures of finasteride drug substance and its degradation products

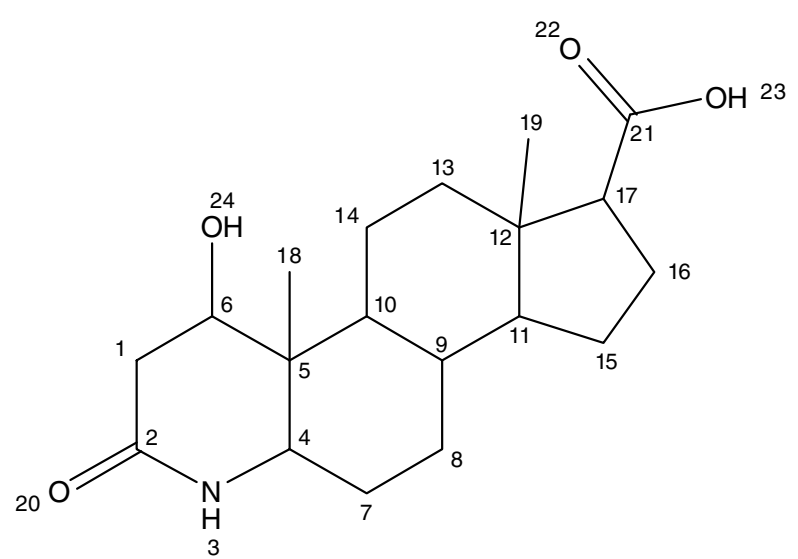

A

DP-1

Confirmed

Chemical Formula: $\mathrm{C}_{19} \mathrm{H}_{29} \mathrm{NO}_{4}$

Exact Mass: 335.2097<smiles>CC12C(CC[C@@H]1C(=O)O)C1CCC3NC(=O)C(O)CC34C1CCC42C</smiles>

B

DP-1

Not matched with NMR data

Chemical Formula: $\mathrm{C}_{19} \mathrm{H}_{29} \mathrm{NO}_{4}$

Exact Mass: 335.2097

Fig. 3. Possible structures of DP-1 


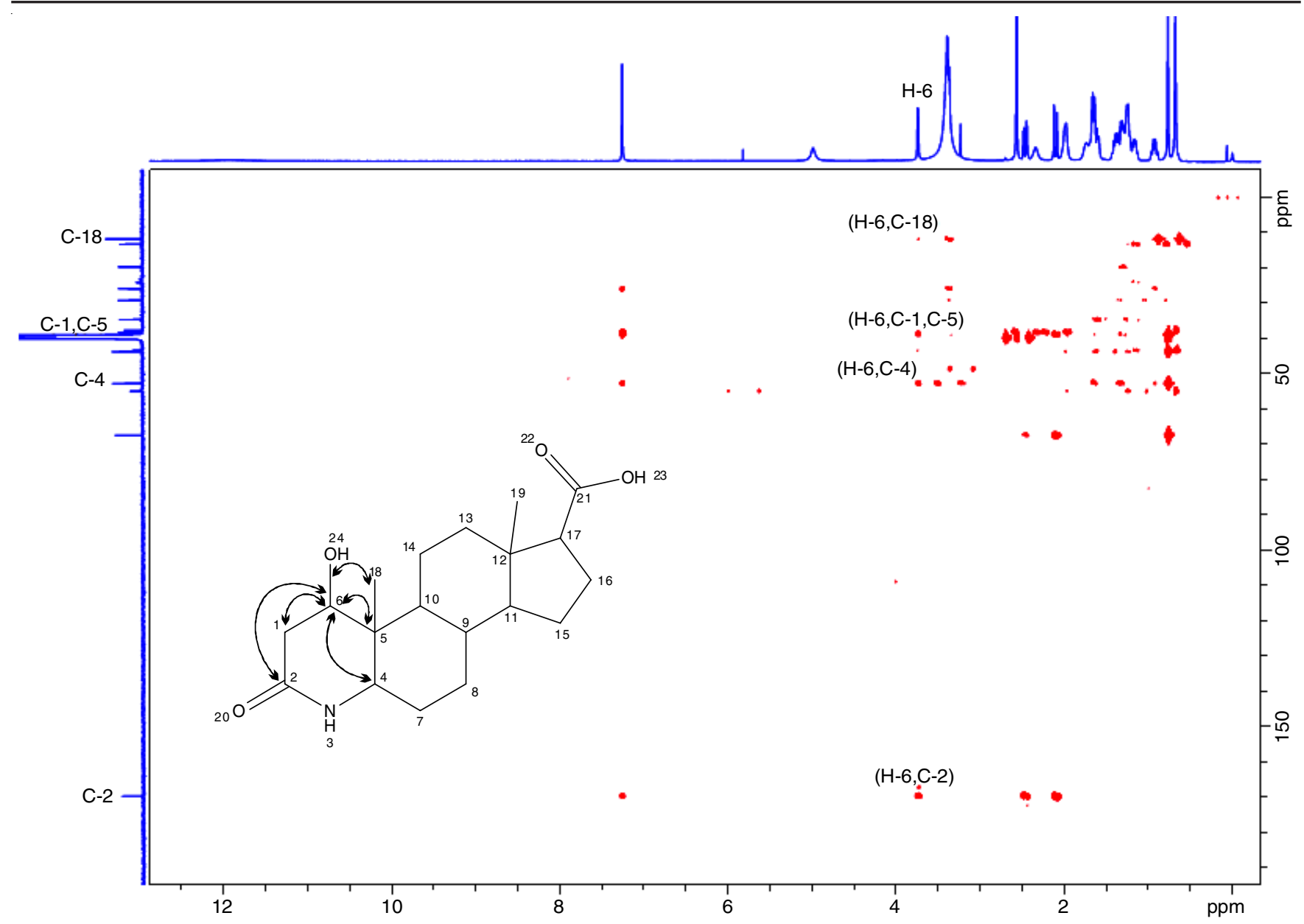

Fig. $4 .{ }^{13} \mathrm{C}$ HMBC spectrum of DP-1

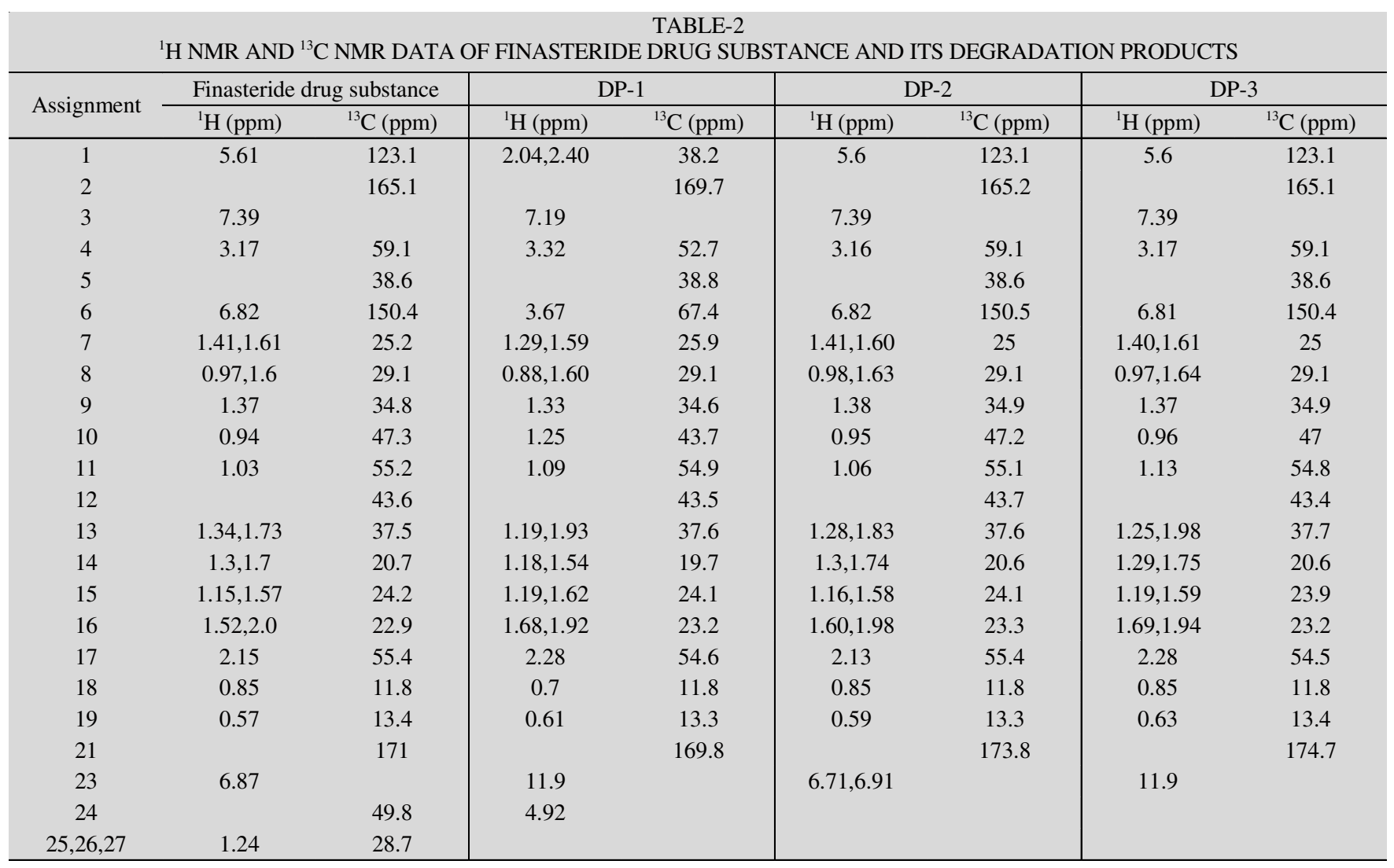


with the structure shown in Fig. 2. The assignment of ${ }^{1} \mathrm{H}$ and ${ }^{13} \mathrm{C}$ chemical shifts for DP-2 were shown in Table-2.

Structure elucidation of DP-3: The HRMS showed protonated molecular ion peak at $\mathrm{m} / \mathrm{z} 318.2074[\mathrm{M}+\mathrm{H}]^{+}$corresponding to m.f. $\mathrm{C}_{19} \mathrm{H}_{27} \mathrm{NO}_{3}$. The ${ }^{1} \mathrm{H}$ NMR of DP-3 had all the protons that are present in finasteride except $\mathrm{NH}$-tertiary butyl group protons. Appearance of a signal for carboxylic acid group at $11.9 \mathrm{ppm}$ indicated the complete hydrolysis of CONHtertiary butyl group to free carboxylic acid under acidic condition. The carbonyl carbon of carboxylic acid was shown at $174.7 \mathrm{ppm}$ in ${ }^{13} \mathrm{C}$ NMR. The analysis of $1 \mathrm{D}$ NMR and 2D NMR data concluded the structure DP-3 shown in Fig. 2. The chemical shift assignment for DP-3 is given in Table-2.

\section{Conclusion}

Forced degradation of finasteride was performed as per the ICH guidelines. The drug was subjected to acid, base and oxidative stress conditions and all the three DPs were found to be formed under acidic stress condition and drug was stable in basic and oxidation conditions. All the three products were separated by prep-HPLC and thoroughly characterized by various 2D NMR and mass spectroscopic methods. The regioselectivity in the formation of DP-1 was confirmed unambiguously. All the three formed degradant products (DP-1, DP-2 and DP-3) are novel as per our literature survey.

\section{CONFLICT OF INTEREST}

The authors declare that there is no conflict of interests regarding the publication of this article.

\section{REFERENCES}

1. T.L. Lemke and D.A. Williams, Foye's Principles of Medicinal Chemistry, Lippincott Williams \& Wilkins, p. 1286 (2008).

2. I. Morton and J.M. Hall, Concise Dictionary of Pharmacological Agents: Properties and Synonyms, Springer Science \& Business Media., vol. 246, 121 (1999).

3. D. Jain and P.K. Basniwal, J. Pharm. Biomed. Anal., 86, 11 (2013); https://doi.org/10.1016/j.jpba.2013.07.013.

4. S. Singh, M. Junwal, G. Modhe, H. Tiwari, M. Kurmi, N. Parashar and P. Sidduri, TrAC Trends Analyt. Chem., 49, 71 (2013); https://doi.org/10.1016/j.trac.2013.05.006.

5. M. Bakshi and S. Singh, J. Pharm. Biomed. Anal., 28, 1011 (2002); https://doi.org/10.1016/S0731-7085(02)00047-X.

6. ICH, Stability Testing of New Drug Substances and Products Q1A(R2), In: International Conference on Harmonization, IFPMA: Geneva (2003).

7. WHO, Draft Stability Testing of Active Pharmaceutical Ingredients and Pharmaceutical Products, World Health Organization: Geneva (2007).

8. CPMP, Note for Guidance on Stability Testing: Stability Testing of Existing Active Substances and Related Finished Products, Committee for Proprietary Medicinal Products, EMEA: London (2002).

9. G. Srinivas, K.K. Kumar, Y.R. Koti Reddy, K. Mukkanti, G.V. Kanumula and P. Madhavan, J. Chem. Pharm. Res., 3, 987 (2011).

10. A.A. Syed and M.K. Amshumali, J. Pharm. Biomed. Anal., 25, 1015 (2001); https://doi.org/10.1016/S0731-7085(01)00385-5.

11. Y.K. Reddy, G.V. Subba Reddy, K.N. Jaya Veera and K.K. Hotha, Am. J. Anal. Chem., 3, 737 (2012); https://doi.org/10.4236/ajac.2012.311098.

12. P.D. Patel, N.I. Surti and U.M. Upadhyay, Int. J. Pharm. Res. Scholars, 4, 221 (2015).

13. Y.K. Reddy, G.V.S. Reddy, K.N.J. Veera and K.K. Hotha, Am. J. Anal. Chem., 3, 737 (2012);

https://doi.org/10.4236/ajac.2012.311098. 